\title{
Muertes por causas violentas y ciclo económico en Bogotá, Colombia: un estudio de series de tiempo, 1997-2006
}

\author{
José Moreno Montoya1 y Ricardo Sánchez Pedraza²
}

Forma de citar Moreno Montoya J, Sánchez Pedraza R. Muertes por causas violentas y ciclo económico en Bogotá, Colombia: un estudio de series de tiempo, 1997-2006. Rev Panam Salud Publica. 2009;26(1):23-30.

RESUMEN Objetivos. Caracterizar las tendencias temporales del número de muertes por causas violentas en la ciudad de Bogotá entre 1997 y 2006 y analizar su posible asociación con los ciclos económicos de la ciudad.

Métodos. Estudio ecológico a partir de las cifras absolutas de muertes violentas registradas entre enero de 1997 y septiembre de 2006 en Santa Fe de Bogotá, Colombia. Se utilizaron modelos ARIMA de series de tiempo trimestrales para caracterizar el comportamiento de las muertes violentas. Como variables explicativas del ciclo económico de la ciudad se utilizaron las series trimestrales del índice de precios al consumidor, la tasa de desempleo y el número total de personas ocupadas; se evaluó su asociación con las series de muertes mediante modelos de función de transferencia.

Resultados. Del total de 36575 muertes por causas violentas en Bogotá, 57,7\% fueron por homicidios y 23,7\% por accidentes de tránsito. Las series de homicidios, suicidios y muertes por accidentes de tránsito mostraron tendencias decrecientes, con un marcado comportamiento estacional en las series de muertes por accidentes de tránsito y suicidios, con picos en los dos últimos trimestres de cada año. Se hallaron asociaciones significativas entre todas las series de muertes por las diferentes causas con al menos una serie económica y en cada caso se identificó el modelo de función de transferencia respectivo.

Conclusiones. Los homicidios constituyen la mayor parte de las muertes por causas violentas y revelan la existencia de patrones temporales en este tipo de muertes. Se encontraron asociaciones significativas entre las muertes por causas violentas y algunas variables económicas que describen el ciclo de la actividad en la ciudad. Se confirmó que diferentes circunstancias socioeconómicas que afectan a una sociedad pueden influir en el nivel de violencia.

Palabras clave Mortalidad, violencia, homicidio, suicidio, accidentes, Colombia.

La mortalidad por causas violentas es un importante indicador del nivel de desarrollo de una sociedad y ha sido objeto de un amplio análisis en la literatura científica, especialmente en la última dé-

\footnotetext{
1 Universidad Nacional de Colombia, Santa Fe de Bogotá, Colombia. La correspondencia se debe enviar a José Moreno Montoya, Calle 53A No. 28-73 sur, Bogotá, Colombia. Correo electrónico: josemorenomontoya@gmail.com

2 Universidad Nacional de Colombia, Santa Fe de Bogotá, Colombia.
}

cada. Como resultado se han identificado importantes asociaciones con factores de riesgo, como el consumo de drogas o alcohol, y ciertos patrones temporales (1), con picos en los períodos vacacionales o festivos (2-4).

También se ha encontrado una asociación entre el estado de la economía de un país y los niveles de violencia en su población (5). Según investigaciones recientes en las que se compararon las tasas de muertes violentas en poblaciones con in- dicadores económicos más o menos favorables (6) y en períodos de crecimiento y decrecimiento económico de sociedades altamente industrializadas $(7,8)$, existe una asociación inversa entre la mortalidad por causas violentas y el estado económico de una sociedad. No obstante, son aún pocos los estudios de este tipo realizados en países en desarrollo y en ellos no se han analizado con suficiente profundidad los patrones temporales de la mortalidad por causas 
violentas y su posible relación con las fluctuaciones de la actividad económica.

En Colombia, diversas investigaciones sobre la realidad de las muertes violentas indican que la capital, Bogotá, es una ciudad menos violenta que otras, como Medellín y Cali (9). Sin embargo, la violencia urbana intencional continúa siendo un problema de primer orden y constituye la primera causa de muerte en la población bogotana. Esta violencia genera no solo pérdidas de capital humano y físico, sino también altos costos económicos en diversos sectores (10).

La elevada violencia en Colombia es un hecho reconocido tanto internamente como en el extranjero y por sus niveles el país se ubica entre los más violentos del mundo. Con 96 homicidios por cada 100000 habitantes en 2003, Colombia se ubicó junto con los Estados Unidos de América entre los países más violentos de la Región $(11,12)$. Ese año en Bogotá se registraron 1158 homicidios, 7,6\% del total nacional. Sin embargo, se debe señalar que en los últimos 10 años se ha observado una pronunciada tendencia a la disminución en el número de homicidios, al igual que en otras formas de muerte violenta, como las accidentales (13).

Los objetivos de la presente investigación fueron caracterizar las tendencias temporales del número de muertes por causas violentas en la ciudad de Bogotá entre 1997 y 2006 y analizar su posible asociación con los ciclos económicos de la ciudad.

\section{MATERIALES Y MÉTODOS}

Se realizó un estudio ecológico mediante el análisis de series de tiempo $(14,15)$ en la población de Bogotá. Esta ciudad, que conforma el Distrito Capital, está dividida en 20 localidades con más de 1200 barrios y es la capital del departamento de Cundinamarca y de Colombia. Ubicada en el centro del país, sobre una meseta de la Cordillera de los Andes a 2600 m sobre el nivel del mar, Bogotá tiene una población de 6776000 habitantes, según el censo de 2005-2006, su densidad demográfica es de 3912 habitantes $/ \mathrm{km}^{2}$ y la cobertura de servicios públicos básicos es aproximadamente de 97\% (16).

\section{Datos de mortalidad}

Se analizaron las cifras absolutas de muertes violentas por trimestre entre enero de 1997 y septiembre de $2006 .{ }^{3}$ Se utilizaron las cifras absolutas por resultar más apropiadas para el análisis de este tipo de muertes (17) y porque Bogotá carece de cifras demográficas trimestrales precisas que hubieran permitido calcular las tasas.

Todos los datos utilizados en el análisis se obtuvieron del Instituto Nacional de Medicina Legal (INML). La notificación de las muertes por causas violentas es un evento obligatorio en Colombia y el INML es la única entidad autorizada para su difusión.

Se consideraron muertes violentas las registradas con alguno de los siguientes códigos, según la Clasificación Estadística Internacional de Enfermedades y Problemas Relacionados con la Salud, en su décima revisión (CIE 10): accidente de tránsito: V02-V99; otro tipo de accidente (no relacionado con el tránsito): W00-W19, W65-W84, X00-X19, X41-X45, X40-X49 y W00-X59; suicidio: $X 60-X 84$ y Y87.0; y homicidio: X85-X99, Y00-Y09 y Y87.1 (18). En el análisis se excluyeron las muertes violentas por causas indeterminadas (códigos Y10-Y34, Y87.2 y Y89.9).

\section{Datos del ciclo económico}

Para valorar los ciclos económicos de la ciudad se utilizaron tres indicadores:

Índice de precios al consumidor. Es el promedio mensual de los precios ponderados de una canasta de bienes y servicios representativa del consumo de los hogares de un país o comunidad (19). Se utilizó el índice trimestral correspondiente a la ciudad de Bogotá, calculado por la Secretaria de Hacienda Distrital de Bogotá.

Tasa de desempleo. Este indicador se construye a partir de los datos de las encuestas de hogares realizadas por el Departamento Administrativo Nacional de Estadísticas (DANE) e indica el tamaño y la estructura de la fuerza de trabajo desempleada o inactiva en la población (20). Se elabora para el país y las siete principales áreas metropolitanas. En general, se expresa como la relación porcentual entre el número de personas que están buscando trabajo y el número de personas que integran la fuerza laboral.

\footnotetext{
3 Los datos correspondían originalmente a series mensuales, sin embargo, se convirtieron en series trimestrales para facilitar la comparación con las series económicas utilizadas.
}

Para la presente investigación se utilizó el indicador correspondiente a la estimación trimestral de la tasa de desempleo total (abierto y oculto) para la ciudad de Bogotá.

Total de ocupados. Este indicador corresponde al número absoluto de personas ocupadas en edad laboral. Según el Banco de la República, este es el mejor indicador independiente de la actividad del ciclo económico a nivel regional (21).

\section{Análisis estadístico}

Para describir las series de tiempo se construyeron modelos autorregresivos integrados de medias móviles (ARIMA) según la metodología propuesta por Box-Jenkins (22). Para este tipo de análisis se ajusta cada serie estudiada a un modelo aditivo en el que intervienen la media de la serie de interés, ponderaciones que toman en cuenta la influencia de los valores pasados sobre el valor actual de la serie analizada y combinaciones lineales de errores aleatorios con una media de cero y variación constante.

En la práctica, son modelos con seis parámetros $(\mathrm{p}, \mathrm{d}, \mathrm{q}, \mathrm{P}, \mathrm{D}, \mathrm{Q})$, donde, $p$ representa el grado de autorregresividad de la serie y verifica la existencia de memoria en el proceso; $d$ es el grado de integración o existencia de tendencia en el fenómeno; y $q$ representa el número de variaciones previas del fenómeno tenidas en cuenta para el ajuste del modelo. Los parámetros P, D y Q tienen significados similares, pero relacionados con comportamientos estacionales. Los parámetros de interés se estimaron mediante técnicas propias de modelos lineales generalizados. Cada uno de esos modelos se evaluó previamente con pruebas de bondad de ajuste e idoneidad (22).

Para evaluar la asociación entre las series de muertes violentas y las series económicas se construyeron modelos con una variable de función de transferencia que de manera general se ajustan a la siguiente fórmula:

$$
Y(t)=V(B) X(t)+N(t)
$$

donde, $Y$ representa el proceso de salida o serie influenciada (muertes violentas), $X$ es la variable explicativa o serie que influye (del ciclo económico), $t$ es el período de tiempo analizado, $V(\mathrm{~B})$ una combinación lineal de valores pasados de $\mathrm{X}, \mathrm{y} N$ representa una combinación de errores aleatorios obtenidos por el mo- 
CUADRO 1. Distribución trimestral de muertes violentas en Bogotá entre enero de 1997 y septiembre de 2006

\begin{tabular}{|c|c|c|c|c|c|c|c|c|c|c|}
\hline & & & & & & & & & & \\
\hline Año/tr & & Total & No. & $\%$ & No. & $\%$ & No. & $\%$ & No. & $\%$ \\
\hline 1997 & 1 & 1143 & 77 & 6,7 & 714 & 62,5 & 247 & 21,6 & 105 & 9,2 \\
\hline & 2 & 1138 & 67 & 5,9 & 732 & 64,3 & 223 & 19,6 & 116 & 10,2 \\
\hline & 3 & 1136 & 94 & 8,3 & 689 & 60,7 & 221 & 19,5 & 132 & 11,6 \\
\hline & 4 & 1107 & 82 & 7,4 & 689 & 62,2 & 239 & 21,6 & 97 & 8,8 \\
\hline 1998 & 1 & 1097 & 104 & 9,5 & 597 & 54,4 & 271 & 24,7 & 125 & 11,4 \\
\hline & 2 & 1146 & 95 & 8,3 & 651 & 56,8 & 293 & 25,6 & 107 & 9,3 \\
\hline & 3 & 1148 & 76 & 6,6 & 646 & 56,3 & 288 & 25,1 & 138 & 12,0 \\
\hline & 4 & 1280 & 107 & 8,4 & 739 & 57,7 & 294 & 23,0 & 140 & 10,9 \\
\hline 1999 & 1 & 1028 & 80 & 7,8 & 593 & 57,7 & 247 & 24,0 & 108 & 10,5 \\
\hline & 2 & 1104 & 91 & 8,2 & 619 & 56,1 & 295 & 26,7 & 99 & 9,0 \\
\hline & 3 & 1180 & 91 & 7,7 & 678 & 57,5 & 262 & 22,2 & 149 & 12,6 \\
\hline & 4 & 1365 & 96 & 7,0 & 793 & 58,1 & 315 & 23,1 & 161 & 11,8 \\
\hline 2000 & 1 & 1083 & 87 & 8,0 & 627 & 57,9 & 255 & 23,5 & 114 & 10,5 \\
\hline & 2 & 1110 & 106 & 9,5 & 638 & 57,5 & 256 & 23,1 & 110 & 9,9 \\
\hline & 3 & 1019 & 92 & 9,0 & 549 & 53,9 & 244 & 23,9 & 134 & 13,2 \\
\hline & 4 & 1053 & 79 & 7,5 & 598 & 56,8 & 271 & 25,7 & 105 & 10,0 \\
\hline 2001 & 1 & 898 & 84 & 9,4 & 470 & 52,3 & 245 & 27,3 & 99 & 11,0 \\
\hline & 2 & 960 & 80 & 8,3 & 576 & 60,0 & 218 & 22,7 & 86 & 9,0 \\
\hline & 3 & 936 & 72 & 7,7 & 550 & 58,8 & 222 & 23,7 & 92 & 9,8 \\
\hline & 4 & 1099 & 82 & 7,5 & 656 & 59,7 & 255 & 23,2 & 106 & 9,6 \\
\hline 2002 & 1 & 886 & 62 & 7,0 & 520 & 58,7 & 196 & 22,1 & 108 & 12,2 \\
\hline & 2 & 888 & 72 & 8,1 & 509 & 57,3 & 208 & 23,4 & 99 & 11,1 \\
\hline & 3 & 995 & 80 & 8,0 & 546 & 54,9 & 249 & 25,0 & 120 & 12,1 \\
\hline & 4 & 875 & 72 & 8,2 & 482 & 55,1 & 231 & 26,4 & 90 & 10,3 \\
\hline 2003 & 1 & 777 & 70 & 9,0 & 419 & 53,9 & 208 & 26,8 & 80 & 10,3 \\
\hline & 2 & 829 & 91 & 11,0 & 457 & 55,1 & 187 & 22,6 & 94 & 11,3 \\
\hline & 3 & 799 & 77 & 9,6 & 434 & 54,3 & 197 & 24,7 & 91 & 11,4 \\
\hline & 4 & 874 & 68 & 7,8 & 460 & 52,6 & 216 & 24,7 & 130 & 14,9 \\
\hline 2004 & 1 & 760 & 65 & 8,6 & 428 & 56,3 & 168 & 22,1 & 99 & 13,0 \\
\hline & 2 & 814 & 60 & 7,4 & 407 & 50,0 & 245 & 30,1 & 102 & 12,5 \\
\hline & 3 & 753 & 60 & 8,0 & 430 & 57,1 & 191 & 25,4 & 72 & 9,6 \\
\hline & 4 & 819 & 57 & 7,0 & 445 & 54,3 & 216 & 26,4 & 101 & 12,3 \\
\hline 2005 & 1 & 753 & 48 & 6,4 & 449 & 59,6 & 179 & 23,8 & 77 & 10,2 \\
\hline & 2 & 724 & 60 & 8,3 & 435 & 60,1 & 155 & 21,4 & 74 & 10,2 \\
\hline & 3 & 715 & 48 & 6,7 & 428 & 59,9 & 188 & 26,3 & 51 & 7,1 \\
\hline & 4 & 731 & 61 & 8,3 & 437 & 59,8 & 172 & 23,5 & 61 & 8,3 \\
\hline 2006 & 1 & 460 & 36 & 7,8 & 310 & 67,4 & 80 & 17,4 & 34 & 7,4 \\
\hline & 2 & 536 & 45 & 8,4 & 345 & 64,4 & 115 & 21,5 & 31 & 5,8 \\
\hline & 3 & 557 & 46 & 8,3 & 362 & 65,0 & 106 & 19,0 & 43 & 7,7 \\
\hline Total & & 36575 & 2920 & 8,0 & 21107 & 57,7 & 8668 & 23,7 & 3880 & 10,6 \\
\hline Prome & & & & & & & & & & \\
\hline trim & & 788 & 75 & & 541 & & 223 & & 100 & \\
\hline
\end{tabular}

Fuente: A partir de datos del Instituto Nacional de Medicina Legal de Colombia.

delo. Este análisis parte de la premisa de que los modelos de este tipo solo se pueden obtener y validar cuando corresponden a series asociadas significativamente, lo que se comprueba mediante la convergencia de los algoritmos de identificación de las funciones empleadas (22). Para calcular adecuadamente el término $V$ se utilizó la metodología propuesta por Chiogna y colaboradores (23); los análisis de bondad de ajuste e idoneidad del modelo fueron los mismos que se utilizaron en el análisis de varianza simple $y$, de manera similar, el modelo se construyó en función de la correlación de las series bajo la premisa de que existía una relación unidireccional entre las series de entrada y de salida. Los modelos elaborados se validaron mediante pruebas estadísticas no paramétricas de aleatoriedad de los residuos del modelo y de su independencia.

Para los análisis se utilizaron los paquetes estadísticos STATA (v. 10.0), SAS (v. 9.1), R (v. 2.07) y X12-ARIMA (24-27).

\section{RESULTADOS}

En total se registraron 36575 muertes por causas violentas especificadas en el período analizado, distribuidas de la siguiente forma: $21107(57,7 \%)$ homici- dios, $2920(8,0 \%)$ suicidios, $8668(23,7 \%)$ por accidentes de tránsito y $3880(10,6 \%)$ por otros accidentes. Los homicidios y las muertes por accidentes de tránsito acumularon $81,4 \%$ del total de las muertes registradas por causas violentas (cuadro 1). Además, hubo 237 muertes por causas violentas no especificadas que no se incluyeron en este análisis.

\section{Series de muertes violentas}

Homicidios. El promedio trimestral de muertes por homicidios en el período estudiado fue de 541. La serie de homicidios mostró una clara tendencia decre- 
FIGURA 1. Representación gráfica de las series de tiempo de muertes por causas violentas por trimestre. Bogotá, de enero de 1997 a septiembre de 2006
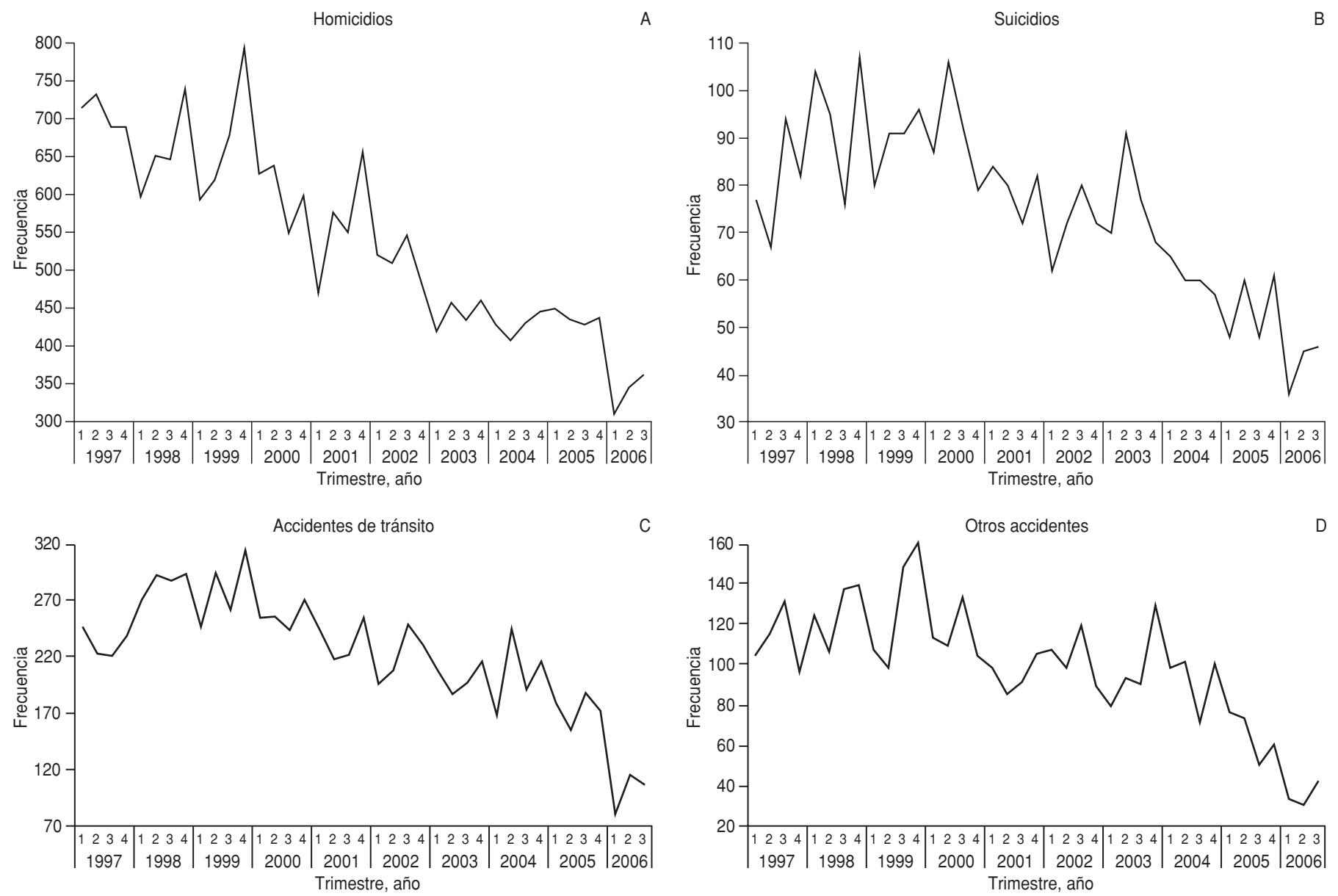

ciente, con un máximo de 793 en el cuarto trimestre de 1999 y un mínimo de 310 en el primer trimestre de 2006 (figura 1A). El modelo ARIMA elaborado a partir de la estructura de la autocorrelación observada para esta serie $(1,1,0)$ —es decir, los valores de trimestres anteriores influyeron significativamente sobre los valores del trimestre analizado- confirmó la existencia de una tendencia decreciente.

Suicidios. El promedio trimestral de muertes por suicidios fue de 75 , con un máximo de 107 en el cuarto trimestre de 1998 y un mínimo de 36 en el primer trimestre de 2006 (figura 1B). Aunque se observó una tendencia a la disminución, esta serie mostró un gran número de picos a lo largo del período analizado. Las características de correlación de la serie mostraron un comportamiento estacional, lo que llevó a un modelo ARIMA del tipo $(1,1,0)(0,1,1) 4$-es decir, que además de los valores de trimestres pasados, los valores ocurridos un año antes influyeron también significativamente en los valores actuales del fenómeno-. Esto confirmó la existencia de la tendencia y mostró que los picos de aumento y disminución en el número de suicidios ocurrían cada cuatro trimestres (anual), lo que indica un comportamiento estacional en la variación del número de suicidios, con máximos en los dos últimos trimestres del año.

Muertes por accidentes de tránsito. En el período analizado, el promedio trimestral de muertes por accidentes de tránsito fue de 223 , con un máximo de 315 en el cuarto trimestre de 1999 y un mínimo de 80 en el primer trimestre de 2006 (figura 1C). Esta serie presentó una clara tendencia decreciente, confirmada mediante la estructura de la autocorrelación observada y el ajuste de un modelo ARIMA $(0,1,1)(0,1,1) 4$, que mostraba también un marcado componente estacional con picos máximos en el cuarto trimestre de cada año.
Muertes por otros accidentes. El promedio trimestral de muertes por accidentes no relacionados con el tránsito fue de 100 , con un máximo de 161 en el cuarto trimestre de 1999 y un mínimo de 31 en el segundo trimestre de 2006. A diferencia de las demás series, en este caso no se observó una tendencia específica ni un comportamiento marcadamente estacional (figura 1D), aunque se aprecia un descenso en el número de muertes por esta causa a partir del año 2003. El modelo ARIMA ajustado $(1,0,1)$ indica que los datos de los períodos previos inmediatos afectaban significativamente el número de muertes en el trimestre siguiente.

\section{Modelos de función de transferencia}

Se encontraron asociaciones significativas entre todas las series de muertes violentas con al menos una de las series del ciclo económico analizadas. En los casos en que se confirmó esa asociación mediante una función simple de transfe- 
rencia (con una sola variable), se elaboró un modelo del comportamiento de la serie de muertes en función de los valores actuales y pasados de las series económicas ajustadas (cuadro 2). Los modelos elaborados tuvieron niveles de ajuste muy bajos durante los primeros trimestres estudiados debido a la escasa información disponible, sin embargo, en los trimestres y años posteriores, las series ajustadas se aproximaron más a la serie real.

Los modelos propuestos cumplieron con los supuestos de bondad de ajuste e idoneidad requeridos para su validez.

\section{DISCUSIÓN}

En esta investigación se caracterizó la mortalidad por causas violentas en la ciudad de Bogotá entre enero de 1997 y septiembre de 2006. Este problema y el desarrollo e implementación de herramientas para su análisis no han contado hasta el momento con la atención suficiente en América Latina.

Se observaron marcadas tendencias decrecientes en el número de homicidios, suicidios y muertes por accidentes de tránsito. Se encontró un comportamiento estacional en las series de muertes por accidentes de tránsito y suicidios, una hipótesis ampliamente discutida en la literatura científica sobre conductas autolíticas (28). A diferencia de las demás series, la de muertes por accidentes no relacionados con el tránsito solamente se vio influida por los eventos similares ocurridos en el trimestre previo, lo que permite descartar una tendencia específica o un patrón más complejo de comportamiento en el tiempo, como la estacionalidad.

El análisis bifactorial y los modelos de función de transferencia confirmaron la asociación significativa entre los patrones de comportamiento de las series de muertes por causas violentas y la dinámica macroeconómica de la ciudad. Series específicas, como la tasa de desempleo, lograron explicar una gran parte de la variabilidad en el tiempo de todas las series de muertes violentas analizadas. El índice de precios sólo mostró asociación con la serie de homicidios y las muertes por accidentes no vinculados con el tránsito, en tanto que el total de ocupados de la ciudad se asoció signifi- cativamente con todas las series de muertes, excepto los suicidios.

La mayoría de los trabajos realizados en los países latinoamericanos reflejan la precariedad de los registros disponibles, aunque consideran, en general, que los registros más confiables son los que recogen las muertes por homicidios, por sus particularidades e implicaciones y por su estrecha relación con la violencia armada (29).

En Bogotá, las muertes por causas violentas constituyen una parte importante de las muertes ocurridas en la ciudad, posiblemente debido a la disminución en el número de muertes por otras causas. Por ello, estudiar este problema a partir de los valores absolutos puede contribuir a develar las tendencias netas sin la influencia de la dinámica de las muertes por otras causas. Por otra parte, aunque en Bogotá diversos programas y acciones - como la restricción en el consumo de bebidas alcohólicas y el uso y porte de armas- han influido en la reducción del problema, aún no se ha analizado el impacto real de esos programas con suficiente rigor metodológico.

$\mathrm{Al}$ analizar los resultados del presente trabajo se deben tener en cuenta algunas limitaciones. En primer lugar, el carácter retrospectivo y ecológico de esta investigación impidió identificar relaciones de causalidad entre los factores analizados; además, la confiabilidad de los resultados depende de la calidad de las fuentes secundarias de información empleadas. Los modelos utilizados, aunque validados, tienen un limitado alcance de predicción, por lo que los resultados deben verse solamente como una aproximación a la comprensión de la estructura temporal que soporta la dinámica del número de muertes por causas violentas. La limitada información disponible en la ciudad impidió tomar en cuenta algunas variables de control que pudieran influir en las muertes violentas y algunos indicadores económicos que pudieran describir mejor la dinámica macroeconómica de la ciudad. Sin embargo, estos resultados están respaldados por el hecho de haber utilizado cifras oficiales provistas por el INML, consideradas confiables y abarcadoras, ya que, como se dijo antes, en Colombia la muerte por causas externas es un evento de notificación obligatoria al INML.
A pesar de estas limitaciones, los resultados de este estudio confirmaron que las muertes por homicidios constituyen la mayor parte de las muertes por causas violentas en Bogotá y revelaron la existencia de patrones temporales en este tipo de muertes. Se encontraron asociaciones significativas entre las muertes por causas violentas y algunas variables económicas que describen el ciclo de la actividad en la ciudad. Aún cuando estos resultados no revelan ninguna relación de causalidad, abren una nueva ventana de análisis sobre la violencia en Bogotá, ya que muestran el comportamiento de este importante problema en función de la dinámica de los eventos macroeconómicos y promueven la inclusión de variables exógenas que podrían asociarse o facilitar la comprensión de la violencia. El análisis realizado confirmó, además, que diferentes circunstancias socioeconómicas que afectan a una sociedad pueden influir en el nivel de violencia.

En análisis futuros se deben incluir variables de control —como variables geográficas, socioeconómicas y sociodemográficas para cada muerte- que permitan profundizar y fortalecer las asociaciones encontradas. Esto es particularmente importante al analizar la mortalidad por causas violentas en grandes urbes, en las que la existencia de varios microambientes urbanos puede generar dinámicas diferentes de violencia. Para incluir variables exógenas se deben refinar los indicadores $\mathrm{o}$, incluso, construir índices generales que reflejen la actividad económica, a fin de incrementar la validez y el alcance de las asociaciones encontradas.

Agradecimientos. Los autores agradecen a Monica Chiogna, Carlo Gaetan y Guido Masarotto por facilitar el algoritmo de identificación de las funciones de transferencia utilizado en el presente proyecto e implementado en el paquete estadístico R. También agradecen al profesor Jorge Martínez, de la Universidad Nacional de Colombia, por su asesoría en el análisis estadístico. Finalmente, se agradece a la Universidad Nacional de Colombia y a su Instituto de Investigaciones Clínicas por garantizar los recursos técnicos necesarios para el desarrollo del proyecto. 
CUADRO 2. Modelos bifactoriales de función de transferencia entre las series de muertes violentas y las series económicas. Bogotá, de enero de 1997 a septiembre de 2006

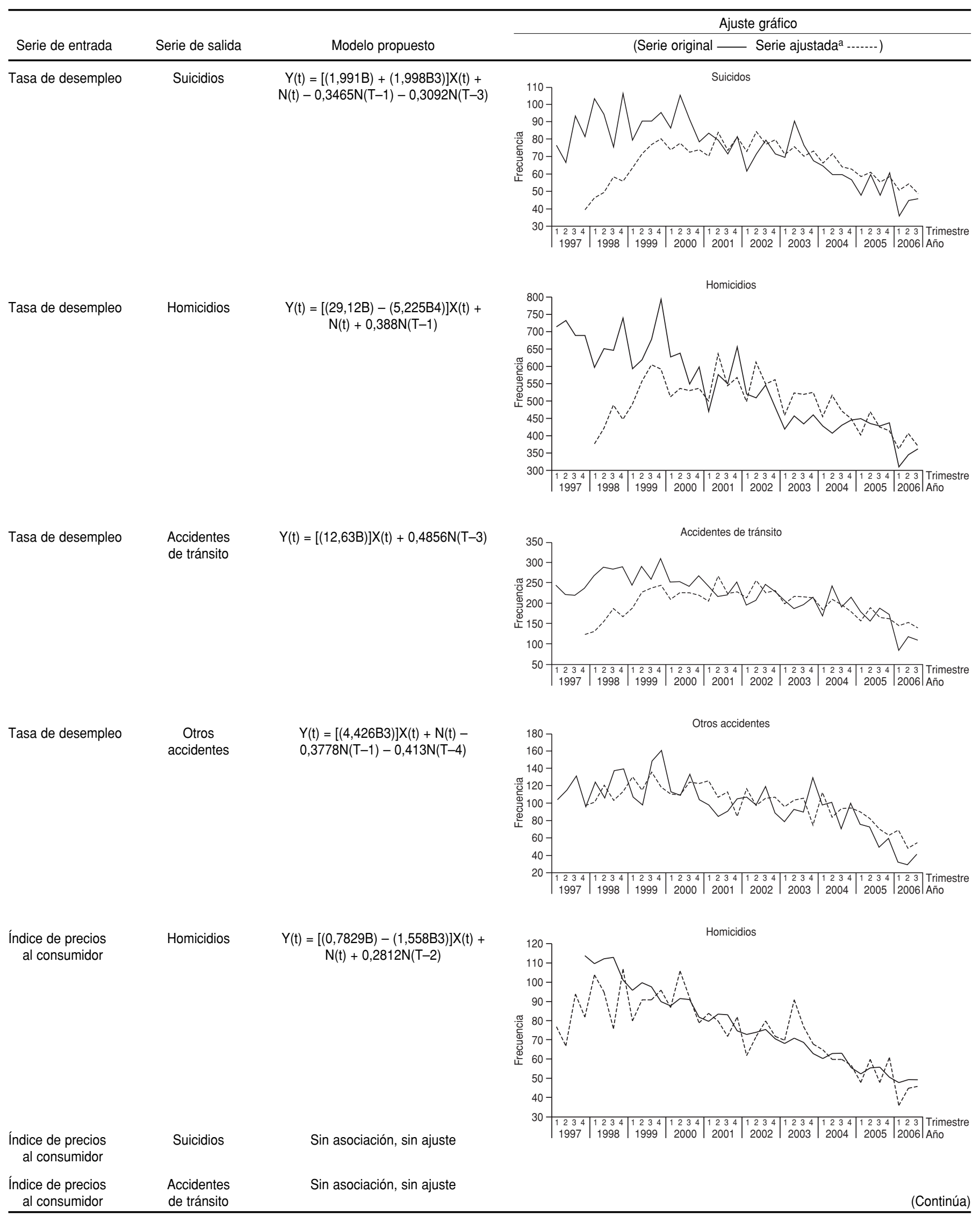




\section{CUADRO 2. (Continuación)}

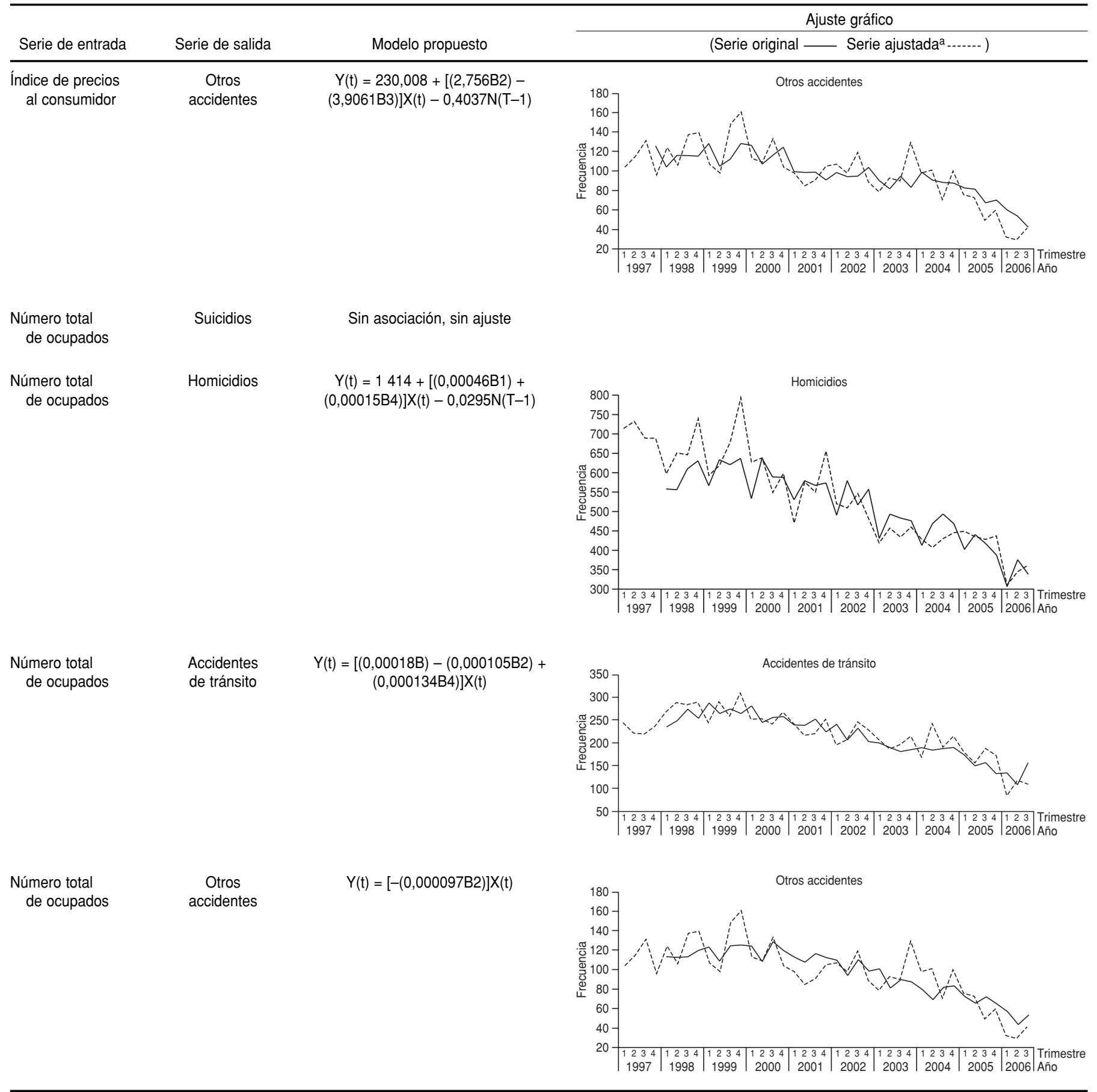

a Para las series ajustadas no se tomaron en cuenta los primeros trimestres por tener un ajuste insuficiente debido a la calidad de los datos.

\section{REFERENCIAS}

1. Sánchez R, Tejada P, Martínez J. Patterns of violent death in Bogotá, 1997-2003. Rev Salud Publica (Bogota). 2005;7(3):254-67.

2. Barata RB, Ribeiro MA, Moraes JC. Tendência temporal da mortalidade por homicídios na cidade de São Paulo, Brasil, 1979-1994. Cad Saude Publica. 1999;15:711-8.

3. Foxcroft DR, Ireland D, Lister-Sharp DJ, Lowe G, Breen R. Long term primary prevention for alcohol misuse in young people: a systematic review. Addiction. 2003;98(4):397411.

4. Degenhardt L, Day C, Hall W, Conroy E, Gilmour $\mathrm{S}$. Was an increase in cocaine use among injecting drug users in New South Wales, 
Australia, accompanied by an increase in violent crime? BMC Public Health. 2005;5:40.

5. Rodríguez F. La pobreza como un proceso de violencia estructural. Rev Cienc Soc. 2004; 10(1):42-50.

6. Herzlich C, Pierret J. De ayer a hoy: construcción social del enfermo. Cuad Med Soc (Rosario). 1989:43:123-34

7. Tardiff K. Patterns and major determinants of homicide in the United States. Hosp Community Psychiatry. 1985;36(6):632-9.

8. Lester D, Motohashi Y, Yang B. The impact of the economy on suicide and homicide rates in Japan and the United States. Int J Soc Psychiatry. 1992;38(4):314-7.

9. Melo J. Cincuenta años de homicidios: tendencias y perspectivas. Razón Pública (Colombia). 2008 octubre 10. Hallado en http:// www.razonpublica.org.co/?p=124\&pdf version=1. Acceso el 7 de mayo de 2009.

10. Duque LF, Klevens J, Ramírez C. Cross sectional survey of perpetrators, victims and witnesses of violence in Bogota, Colombia. J Epidemiol Community Health. 2003;57:355-60.

11. Gaitán F, Díaz J. The violence in Colombia: some basic explanations. En: Concha Eastman A, Carrión F, Cobo G, eds. Cities and violence in Latin America. Quito: United Nations Urban Management Program, Regional Office for Latin America and the Caribbean; 1994. Pp. 75-9. (Urban Management Series No. 2.)

12. US Department of Justice. Uniform crime report, 2003. Washington, D.C.: Department of Justice, Federal Bureau of Investigation; 1992.
13. Secretaría de Gobierno de Bogotá. Marzo, el mes más seguro en los últimos 10 años en Bogotá. Bogotá: Secretaría de Gobierno de Bogotá; 2008. Hallado en http://www.gobierno bogota.gov.co/content/view/168/337/. Acceso el 7 de mayo de 2009.

14. Morgenstern H. Ecologic studies. En: Rothman K, Greenland S, ed. Modern epidemiology. Filadelfia: Lippincott Raven; 1998. Pp. 459-80.

15. Morgenstern H. Uses of ecologic analysis in epidemiologic research. Am J Public Health. 1982;72(12):1336-44.

16. Departamento Administrativo Nacional de Estadística. Datos oficiales sobre Bogotá, resultados del Censo 2005-2006. Bogotá: DANE; 2007.

17. Abramson JH, Abramson ZH. Making sense of data. A self-instruction manual on the interpretation of epidemiological data. 3rd ed. New York: Oxford University Press; 2001. Pp. 10.

18. World Health Organization. International statistical classification of diseases and related health problems. 10th revision, version for 2006. Geneva: WHO; 2006.

19. Departamento Administrativo Nacional de Estadística. Índice de precios al consumidor. Ficha metodológica. Bogotá: DANE; 2007.

20. Departamento Administrativo Nacional de Estadística. Gran Encuesta de Hogares. Ficha metodológica. Bogotá: DANE; 2007.

21. Zuccardi IE. Los ciclos económicos regionales en Colombia. Documento de trabajo sobre economía regional. Bogotá: Banco de la República de Colombia; 2003.
22. Brocwell $\mathrm{P}$, Davis J. Introduction to time series and forecasting. Springer text in statistics. 2nd ed. New York: Springer Verlag; 2004. Pp. 78-92.

23. Chiogna M, Gaetan C, Masarotto G. Automatic identification of seasonal transfer function models by means of iterative stepwise and genetic algorithms. J Time Ser Anal. 2007;(29)1: 37-50.

24. Stata Corporation. Stata/IC 10.0 for Windows. College Station, TX: Stata Corp.; 2008.

25. SAS Institute. The SAS System for Windows, version 9 TS. Cary, NC: SAS Institute; 2002.

26. The $R$ Foundation for Statistical Computing. $R$ version 2.07. Vienna: The R Foundation; 2008.

27. US Census Bureau. X12-Arima. Washington, D.C.: US Census Bureau; 2008.

28. Sánchez R, Orejarena S, Guzmán Y. Características de los suicidas en Bogotá: 1985-2000. Rev Salud Publica. 2004;6(3);217-34.

29. Cardona M, García I, Giraldo A, López M, Suárez C, Corcho D, et al. Homicides in Medellín, Colombia, from 1990 to 2002: victims, motives, and circumstances. Cad Saude Publica. 2005;21(3):840-51.

Manuscrito recibido el 14 de mayo de 2008. Aceptado para publicación, tras revisión, el 9 de diciembre de 2008.
ABSTRACT

\section{Death from violent causes and economic cycles in Bogota, Colombia: a time-series study, 1997-2006}

Key words
Objectives. To determine seasonal trends in the number of deaths from violence in Bogotá, Colombia from 1997-2006 and to analyze any possible associations with the city's economic cycles.

Methods. An ecologic study based on the official death toll from violence registered between January 1997 and September 2006 in Santa Fe de Bogotá, Colombia. Auto Regressive Integrated Moving Average (ARIMA) time-series trimester models were used to describe the behavior of violent deaths. The explanatory variables for the city's economic cycles were the trimester series of the consumer price index, the unemployment rate, and the total number of people employed; these were related to the death series through transfer function models.

Results. Of the 36575 total deaths from violence in Bogotá, 57.7\% were homicides and $23.7 \%$ resulted from traffic accidents. The homicide, suicide, and traffic accident deaths showed downward trends, with a noteworthy seasonal pattern in the traffic accident and suicide deaths that peaked during the last two trimesters of each year. Significant associations were found between all the death series from all the different causes and at least one economic series, and in each case a respective transfer function model was identified.

Conclusions. Homicides constituted the largest portion of the violent deaths, and exposed a seasonal pattern to this death type. Significant associations were found between deaths from violence and some economic variables, revealing a cycle tied to the life of the city. The socioeconomic circumstances affecting a society were confirmed to influence the level of violence.

Mortality, violence, homicide, suicide, accidents, Colombia. 